\title{
Changes in expression of genes associated with neurogenesis during development of Alzheimer's disease signs in OXYS rats
}

\author{
Burnyasheva A.O.*, Rudnitskaya E.A., Stefanova N.A., Kolosova N.G. \\ Institute of Cytology and Genetics SB RAS, Novosibirsk, Russia \\ *e-mail: burn.alena2505@mail.ru
}

Key words: neurogenesis, neurotrophin, Alzheimer's disease, OXYS rats

Motivation and Aim: Alzheimer's disease (AD) is the most common type of age-related dementia worldwide. However, the precise mechanisms of its pathogenesis are not fully understood. One of the processes that may contribute to neurodegeneration is alteration of neuronal plasticity. Neurogenesis is one of the major mechanisms of neuronal plasticity, and neurotrophic supply is crucial for it. Using OXYS rats as a suitable model of $\mathrm{AD}$ previously we have shown that development of $\mathrm{AD}$ signs is accompanied by changes in expression of genes involved in neurotrophic signaling pathway. Thus in this work we investigated a link between changes in expression of neurogenesis-associated genes and development of AD-like pathology in OXYS rats.

Methods and Algorithms: 20-days-, 3-5- and 18-months-old male OXYS and Wistar (control) rats were used. The RNA-seq data obtained for hippocampus were used to analyze differentially expressed genes involved in neurogenesis according to MANGO (Mammalian Adult Neurogenesis Gene Ontology) database. These genes were functionally annotated using DAVID (Database for Annotation, Visualization and Integrated Discovery). ELISA was used to quantify the levels of nerve growth factor (NGF) and brainderived neurotrophic factor (BDNF), TrkA and $\mathrm{p} 75^{\mathrm{NTR}}$ receptors, western-blot analysis was used to quantify levels of TrkB and phosphorylated TrkB (phTrkB) receptors in the hippocampus.

Results: We found that to the age of manifestation of AD signs in OXYS rats (from 20 days to 5 months) changes in expression of 22 genes were involved in the hippocampal neurogenesis. Functionally, these genes were associated with neuronal precursor cell proliferation, cellular response to glucose stimulus and positive regulation of angiogenesis. Changes in gene's expression might be due to activation of neurotrophic signaling observed in OXYS rats at the age of 3 months: levels of BDNF, pro-survival TrkA receptor of NGF and pro-apoptotic $\mathrm{p} 75^{\mathrm{NTR}}$ receptor were higher compared to Wistar rats. Progression of AD-like pathology in OXYS rats (from 5 to 18 months of age) is accompanied by changes in expression of 25 genes involved in neurogenesis. These genes were associated with angiogenesis, response to oxidative stress and negative regulation of cell death. The changes occurred against background of depletion of neurotrophic supply: the levels of BDNF and activation of its receptor (phTrkB/TrkB ratio) decreased in OXYS rats. However, the levels of NGF increased with age in both rat strains and may be considered as a compensatory response to slow down neuronal loss of function.

Conclusion: Alterations of neurotrophic supply in the hippocampus occur during development of AD-like pathology in OXYS rats and may result in disturbances of hippocampal neurogenesis.

Acknowledgements: This work was supported by grant from the Russian Science Foundation (project \# 1915-00044). 